\title{
Survey of Ankle Joint Sport Injury and Research of Rapid Rehabilitation Method
}

\section{Y.Q. ZHANG}

Department of Physical Education, Xi'an Shiyou University, China

\begin{abstract}
The ankle joint has important significance in maintaining body weight and motor functions. If the ankle joint is not handled properly after injury, a variety of complications can be easily produced. In the paper, it is found that ankle joint injury has higher proportion through questionnaire survey. Clinical verification shows that ankle joint sport injury is treated by special techniques with significant effect and short treatment effect. Patients are willing to accept. It is proposed that prevention of ankle joint sport injury should be focused in PE professional teaching and training.
\end{abstract}

KEYWORD: Ankle joint; Sport injury; Treatment; Rapid rehabilitation

\section{INTRODUCTION}

Ankle joint injury is the most common injury in sports, which accounts for more than $8 \%$ in the whole sport injuries. Patients can suffer from local swelling, pain, congestion and limp after ankle joint injury. If the injured part is not properly handled, injury is aggravated, rehabilitation period is prolonged, and even sequelaes can be produced. Normal implementation of sports teaching, sports training and sports competition is directly affected. Such injury can improve sports achievements and shorten sports life of professional athletes. Adverse psychological influence can be produced on athletes, etc. Then, prevention of ankle joint injury, rapid treatment rehabilitation and complication reduction after injury are important topics worthy of study in sports teaching and training.

\section{A SURVEY OF ANKLE JOINT SPORT INJURY}

\subsection{Respondents and Methods}

\subsubsection{Object}

120 amateur gymnastics in colleges and universities majoring sports from 2009 to 2010 were included, wherein 59 athletes accepting treatment due to sport injury were regarded as research objects, including 54 male athletes and 66 female athletes with age of 15-26 years old. Average age of male athletes was $(19.4 \pm 2.9)$ years old, and average age of female athletes was $(20.1 \pm 2.4)$ years old.

\subsubsection{Method}

Self-made questionnaire is adopted, ankle joint sport injuries of respondents in recent one year were collected. Main contents of questionnaire items include gender, age, training programs, training age, injury sites, type, reason, etc. A total of 120 sport injury questionnaires were released to students, 120 questionnaires were valid with recovery rate of $100 \%$. All recovered questionnaires were real and effective according to examination.

Statistical Analysis

Count data underwent chi-square test analysis by using SPSS 13.0 statistical software, and multiple linear regression is adopted for analyzing risk factors affecting injury.

\subsection{Results}

\subsubsection{Injury Incidence}

Incidence of injury: A total of 59 among 120 respondents suffered from ankle joint injury. Total incidence was $49.2 \%$ in total. Wherein a total of 26 male athletes had ankle joint injury with injury incidence of $48.1 \%$ and a total of 33 female athletes had ankle joint injury with injury incidence of $50.0 \%$.

Table 1. Basic Situation of Respondents

\begin{tabular}{|c|c|c|c|c|}
\hline Group & $\mathrm{n}$ & $\begin{array}{c}\text { Age } \\
\text { (Years Old) }\end{array}$ & $\begin{array}{c}\text { Training } \\
\text { Duration }\end{array}$ & $\begin{array}{c}\text { Ankle Joint Injury } \\
\text { (Incidence) }\end{array}$ \\
\hline Female & 54 & $19.4 \pm 2.9$ & $9 \pm 4$ & $26(48.1)$ \\
\hline Male & 66 & $20.1 \pm 2.4$ & $8 \pm 5$ & $33(50.0)$ \\
\hline Total & 120 & $20.1 \pm 2.5$ & $9 \pm 5$ & $59(49.2)$ \\
\hline
\end{tabular}




\subsubsection{Injury Incidence Condition}

59 patients with ankle joint injuries respectively included 18 cases in malleolus medialis, 26 cases in external malleolus and 15 cases in hock with incidence rates of $30.5 \%, 44.1 \%$ and $25.4 \%$. 26 patients were male, including 7 cases of malleolus medialis, 13 cases in external malleolus and 6 cases in hock with incidence rates of $26.9 \%, 50.0 \%$ and 23.1\%. 33 patients were female, including 11 cases in malleolus medialis, 13 cases in external malleolus and 9 cases in hock with incidence rates of $33.3 \%$, $39.4 \%$ and $27.3 \%$. External malleolus has the highest incidence among ankle joint injury, namely $44.1 \%$ followed by malleolus medialis with incidence of $30.5 \%$ and hock with incidence of 25.4\%. Please see Table 2.

Table 2. Respondent Injury Incidence Condition

\begin{tabular}{|c|c|c|c|c|}
\hline Group & $\mathrm{n}$ & $\begin{array}{c}\text { Malleolus medialis } \\
\text { (Incidence) }\end{array}$ & $\begin{array}{c}\text { External malleolus } \\
\text { (Incidence) }\end{array}$ & $\begin{array}{c}\text { Hock } \\
\text { (Incidence) }\end{array}$ \\
\hline Female & 26 & $7(26.9)$ & $13(50.0)$ & $6(23.1)$ \\
\hline Male & 33 & $11(33.3)$ & $13(39.4)$ & $9(27.3)$ \\
\hline Total & 59 & $18(30.5)$ & $26(44.1)$ & $15(25.4)$ \\
\hline
\end{tabular}

\subsection{Analysis}

The main causes of injury in respondents included movement disorders include technical error, the local burden is too heavy and tired, accounting for $18.1 \%$, respectively, $25.0 \%$ and $23.2 \%$. Show gymnasts during training and competition, as a result of skilled movement error, local overburden as well as poor muscle stretching, strong viscosity, small joint range of motion caused by fatigue, thereby finally resulting in injury [1-2].

\section{STUDY OF RAPID REHABILITATION METHOD OF ANKLE JOINT SPORT INJURY}

\subsection{Conventional Treatment of Ankle Joint Injury}

Ankle joint injury is treated according to the following principles: movement limitation, swelling decreasing, pain relieving and functional exercise. Patients should be immediately sent to hospital for corresponding treatment in case of fractures, etc.

Movement Limitation: movement should be immediately stopped, and the injured part should be pressurized and bandaged. The injured limbs should be raised, thereby the injured tissues should rest adequately, thereby reducing tissue bleeding and swelling. Corresponding movement limitation methods should be adopted according to injury conditions, movement should be stopped for patients with slight injury, who can recover through resting for 7 days to 1 day. The injured limbs should be fixed for 10 to 14 days with bandage or tape aiming at patients with serious injury. Corresponding examination should be implemented for serious patients, and the limbs should be fixed with plaster for 4 to 6 weeks after restoration in case of fracture or dislocation.

Swelling Decreasing and Pain Relieving: local icing or cold compression is adopted immediately after injury, thereby significantly reducing or stopping pain. Vessels can be contracted locally, leakage, bleeding, swelling and pain can be reduced by cold compression. Local hot compression, TCM external application and fumigation can be adopted for 1 to 2 days after injury, thereby decreasing swelling, relieving pain, promoting seepage absorption, and accelerating recovery. Drug is locally applied. Meanwhile, anti-inflammatory pain easing drugs can be orally taken. Yunnan Baiyao Aerosol can be sprayed locally, or TCM cape jasmine can be smashed and added with borneol and vinegar, and the mixture can be applied externally, or Voltaren latex can be applied externally aiming at patients with slight injury. Trauma lotion can be used for fumigation in addition to the above methods aiming at patients with serious injury. For example, people with intra-articular hemorrhage should firstly accept puncture for sucking blood. Then, mixture of lidocaine and aristocort or other glucocorticoid can be injected for relieving adhesions. The patients can undergo acupuncture, manipulation and massage therapy in late stage, thereby promoting recovery of joint function.

Function Exercise: joint function exercises can be strengthened during recovery period, power of ligaments and tendon around joints can be reinforced, and the methods have important significance for recovering and reinforcing stability of ankle joints. The period is divided into two stages concretely. Stage 1: since the injured ankle joint generally leads to limitation of dorsiflexion. Various fibula muscle strength is weak accompanied by ankle joint instability and pain. Therefore, dorsiflexion movements without heavy burden and achilles tendon stretching exercises should be strengthened. Activity intensity should be gradually increased. If there are no pain and swelling after activity, the patients can enter exercise in the second stage after dorsiflexion is close to normal stage. Muscle exercise should be continuously implemented in the stage. Ankle joint sport coordination should be reconstructed, weightbearing exercise and walking can be started, which can be gradually transitioned to running. '8'-shaped running should be carried out, thereby training jump until one-foot jump is implemented. Entire period should proceed in an orderly way and step by step, intensity of training should be gradually increased, protective gear can be worn at the start for preventing secondary injury. The protective gear can be removed after muscle strength is stronger[3]. 


\subsection{Clinical Validation}

Case Selection: 60 patients accepting ankle joint injury treatment in physical therapy room at Hunan Institute of Humanities from March 2006 and December 2007 were selected. 60 patients with ankle joint injury were divided into conventional group and special group with 30 cases in each group.

Grouping principle: patients undergoing cold compression treatment before treatment in physical therapy room should be arranged in conventional group, and the other patients were arranged in special group.

Treatment method: Conventional group: the patients should accept cold compression immediately after being injured, and injured limbs should be raised, pressurized and bandaged. Drugs for decreasing swelling, relieving pain and removing blood stasis should be applied externally 1-2 days later. Cold and hot alternating water spa should be adopted 2.0 to 3.0 days later for twice or three times a day. Swelling can be decreased one to two weeks later. Acupuncture therapy should be used in early and middle stages of injury. Corresponding points should be punctured for treatment, and each therapy should last for 15-20 min once a day. Herbal hot compress or fumigation (mainly including medicinal herbs) should be adopted for late stage of injury once a day. Massage should be implemented for 10-15 min then. The injured parts can be healed up within $10 \mathrm{~d}$. Special group: special technique refers that a set of methods for rapidly treating and recovering ankle joint injury completely by hand techniques (including touch, restoration, massage, acupuncture and other integrated approaches) can be summarized through long-term diagnosis treatment practice. It is required that patients should be immediately diagnosed and treated after ankle joint injury. Hand techniques should be immediately implemented under the condition of no fracture and rib breaking in diagnosis. Firstly, patients should sit straightly, feet of patients should be placed flatly, operator should gently massage for 5 to $10 \mathrm{~min}$ on both ends of injured parts. Note: the massage direction should be dispersed opposite to the injured parts for preventing the injured parts from bleeding and swelling. Patients with swelling can produce blood pressure difference by hand technique, thereby returning blood in injured parts, and immediately decreasing swelling. The injured parts should be reset, and grains should be straightened out by gentle touch method. Note: forcible hand technique should not be adopted for producing huge pain on patients and tension in the injured parts. It is difficult to restore the injured parts. The aspect is very important. Namely, it is key for rapid recovery. When doctors implement restoration hand techniques, after the part is touched accurately, water can be 'sprayed' on the injured part for dispersing attention of patients, thereby rapidly restoring the injured parts. Injured parts can be slightly pressed for searching the most painful points. Meridian gas stasis blocking point can be identified, the painful points can be gently pressed immediately with finger tip or palm root. Vibration shaking (internal strength) method should be adopted to push for several minutes opposite to meridian running direction. Tight pushing and slow movement should be implemented, the hand should be pushed for three times and returned once until the painful points are basically eliminated. Pain can disappear immediately once blood vessels are penetrated by pushing according to TCM theory 'no pain for healthy person, and painful person is not penetrated in meridians'. Many patients can be cured in one time.

A set of foot and ankle gymnastics for middle and late stages can also be designed specifically in order to ensure that normal sports function can be recovered after ankle joint injury, improve ankle joint stability, and prevent customary injury in the future, thereby promoting rapider recovery of ankle joint functions. It is required that patients can practice foot and ankle gymnastics once to three times each day. Results: 26 cases were included for analyzed in the conventional group since four documents were not provided. Healing refers to basic decreasing of swelling without pain, and normal activity function was restored. Treatment effect comparison of the two groups is shown in table 3 .

Table 3. Treatment Effect Comparison of Two Groups (n/\%)

\begin{tabular}{|c|c|c|c|c|}
\hline Group & $\mathrm{n}$ & $\begin{array}{c}\text { Healing by } \\
\text { Once to } \\
\text { Three Times }\end{array}$ & $\begin{array}{c}\text { Healing by } \\
\text { Four Times to } \\
\text { Seven Times }\end{array}$ & $\begin{array}{c}\text { Healing by } \\
\text { More than } \\
\text { Eight Times }\end{array}$ \\
\hline $\begin{array}{c}\text { Conventional } \\
\text { Group }\end{array}$ & 26 & 0 & $5 / 19$ & $21 / 81$ \\
\hline Special Group & 30 & $5 / 17$ & $21 / 70$ & $4 / 13$ \\
\hline
\end{tabular}

Table 3 illustrates that no patient can be healed by once to three times, five patients can be healed by four times to seven times accounting for 19\%, 21 cases can be healed by more than 8 times accounting for $81 \%$ in conventional group. Five cases can be healed by once to three times accounting for 17\%, 21 cases can be healed by four times to seven times accounting for $70 \%$ and only four cases can be healed by more than eight times accounting for $13 \%$. Note: treatment effect of special hand technique is prominently better than conventional therapy.

Experiment showed that special hand techniques can be adopted for treating ankle joint sports injury. They break through the concept of traditional routine therapy 'cold compression must be implemented after injury, and message can be adopted $48 \mathrm{~h}$ later'. The treatment effect is prominent compared with conventional treatment method with short healing time. New pain can not be caused on patient, and patients are willing to accept[4-6]. 


\section{PREVENTIVE MEASURES AND RECOMMENDATION}

More and more attention is paid to ankle joint in sports. Training on weak part of ankle joint should be firstly strengthened in teaching and training, training on foot eversion muscle group and ligaments should be particularly strengthened. Foot ankle strength can be enhanced through hooking kettlebells by foot tip, lifting heel with weight, physical therapy instrument, other resistance exercise, shaking plate exercise or massive shaking exercise on balance beam, thereby improving joint stability. Flexibility of ankle joint can be developed by foot injured flexion kneeling (back-roll), trampling disk device for exercises, etc. Some people believe that dash sports achievements can be improved through ankle joint flexibility exercise [79]. Secondly, basic skill training of various specialized sports should be strengthened. Coaches should follow training principle of proceeding in an orderly way and step by step for scientific training. Formation and quality of preparatory activities should be focused according to training and competition. Students should allow students to master necessary self-protective methods in accordance with special characteristics, hidden danger of various sport injuries can be eliminated, athletes with ankle joint injury experience can fix ankle joint by elastic bandage, ankle protector, etc. during training or competition for preventing sports injury. Furthermore, students should be guided for improving awareness on ankle joint injury, medical supervision should be strengthened, excellent idea quality should be cultivated, fair and rational competition is promoted, habits of participating in competition in a civilized mode are formed, unfavorable factors of ankle joint injury should be reduced, and risks of ankle joint sport injury can be lowered.

When sport injuries of ankle joints are treated, the patients should be immediately restored or surgically treated in case of dislocation and fracture. Icing or cold compression should be implemented firstly for common twist and contusion. The injured part should be pressurized or bandaged by 'U'-shaped bandage pad or other forms. Meanwhile, early fixation is combined for treatment, swelling caused after ankle joint twisting can be eliminated to the minimum level, recovery time can be shortened, patients applicable to post-injury recovery and ankle joint twisting injury experience can be treated by inflatable splint ${ }^{[9]}$. Joint position can be corrected, recovery of twisted tissues can be promoted, swelling elimination can be better adapted to splint. Patients with mild sprain can accept rehabilitation training 24 hours later according to different cycles. Massage, brittle ankle acupuncture, ankle eversion and ankle drawing functions recovery training also can be combined so that ankle joints of patients can be timely and effectively treated, and the athletes can start training and competition immediately. It is recommended that the ankle joint should undergo cold compression treatment after each large-load training or competition aiming at characteristics of ankle joint sport injury. The method has the functions reducing blood flow around the joint, anaesthetizing joints, lowering tension on muscle, ligament and fascia, etc. Meanwhile, ankle joint protection supporting belt can be worn for effectively preventing and reducing continuously increased incidence of ankle joint sports injury. Awareness on ankle joint sport injury prevention and health care should be improved, sports facilities should be perfected, the maintenance and management of sports venues should be improved, coaches should focus on systematic and scientific training and teaching, ankle joint function exercise can be strengthened by athletes during comprehensive body exercises. Flexibility and stability of ankle joint can be improved. Medical supervision should reinforced, rehabilitation and health care treatment methods should be mastered, mental state and body level of the athletes should be adjusted, various predisposing factors for causing ankle joint injury should be eliminated, thereby reducing ankle joint sport injury, improving students' physical fitness and health, and creating excellent athletic performance.

\section{REFERENCES}

[1] Zhou Hongmei. Study of Ankle Joint Injury and Treatment in Sports Process. Modern Preventive Medicine, 2011, 20: 42174218.

[2] Cai Kaiming. Exploration of Ankle Joint Injury Causes and Fast Rehabilitation Method. Chinese Tissue Engineering Research and Clinical Rehabilitation, 2009, 13 (28):55675570.

[3] Chen Yong. Analysis of Influence of Ankle Joint Flexibility on Sprint Performance. Sichuan Sports Science, 1991, (1): 36-37.

[4] Xian Hanzhao, Wang Weiping. Inflatable Splint for Handling Ankle Joint Sports Injury. China Sport Science and Technology, 1987 (4), 33-36.

[5] Yang Cheng; Investigation and Research of Ankle Joint Sport Injury for Students in Sports Department. Chongqing University Journal (Social Sciences Version), 1998, (02): 8284.

[6] Mattacola C. G, Dwyer MK. Rehabilitation of the ankle after acute sprain or chronic instability. Journal of Athletic Training, 2002.

[7] Arnold B. L, Docherty C. L. Bracing and rehabilitation-what's new. Clinics in Sports Medicine, 2004.

[8] Hupperets M. D, Verhagen E. A, Mechelen W. The 2BFit study: is an unsupervised proprioceptive balance board training programme, given in addition to usual care, effective in preventing ankle sprain recurrences. Design of a randomized controlled trial. BMC Musculoskelet Disord, 2008.

[9] Hosea T. M, Carey C. C, Harrer M. The gender issue: epidemiology of ankle injury in athletes who participate in basketball. Clinical Orthopaedics and Related Research, 2000. 